\title{
RELA Fusion Positive
}

National Cancer Institute

\section{Source}

National Cancer Institute. RELA Fusion Positive. NCI Thesaurus. Code C156758.

An indication that expression of a fusion containing sequences from RELA has been detected in a sample. 